\title{
ANÁLISE ESPACIAL DE FRAGMENTOS FLORESTAIS COM OCORRÊNCIA DE JARACATIÁ NO NORTE DO PARANÁ - BRASIL
}

\author{
Space analysis of forest fragments \\ with occurrence of Jacaratiá in \\ the North of the State of \\ Paraná - Brazil
}

\author{
Kleber TRABAQUINI ${ }^{1}$ \\ Édison MIGLIORANZA ${ }^{2}$ \\ Valmir de FRANÇA ${ }^{3}$ \\ Ana Odete Santos VIEIRA 4
}

\begin{abstract}
RESUMO
Este trabalho analisa o contexto espacial dos fragmentos onde houve o registro de Jacaratia spinosa (Aubl.) A.DC e avalia a estrutura populacional dessa espécie. Foram analisados sete fragmentos florestais, através do uso do sensoriamento remoto e campanhas de campo. Além disso, realizaram-se avaliações referentes à população e os resultados demonstram que esta está sujeita à ação de perturbações, principalmente antrópicas, o que indica uma alta susceptibilidade à extinção local da espécie caso ocorra morte ou corte desses indivíduos, pois os valores da Densidade Absoluta (DA) e da Dominância Absoluta (DoA) são valores muito baixos quando comparados com outros estudos fitossociológicos.
\end{abstract}

\section{Palavras-chave:}

Caricaceae; distribuição geográfica; espécie nativa.

\begin{abstract}
The present work examines the global context of fragments where Jacaratia spinosa (Aubl.) A.DC has been registered and evaluates the structure of this species population. The land use of seven forest fragments were analyzed through the use of remote sensing and field campaigns. Moreover, there have been assessments regarding population and the results show that they are subject to the anthropogenic disturbance, indicating a high susceptibility to local extinction of the species if death or cutting occur because the values of the Absolute Density (AD) and Absolute Dominance (DoA) are very low when compared to other phytosociological studies.
\end{abstract}

\section{Key words:}

Caricaceae; geographic distribution; the native species.

\footnotetext{
1 Mestrando em Agronomia da Universidade Estadual de Londrina (UEL) e bolsista da Capes. E-mail: klebertrabaquini@yahoo.com.br. 2 Prof. Dr. do Depto. de Agronomia da UEL. Bolsista Produtividade CNPq. E-mail: emiglior@uel.br.

3 Prof. Dr. do Depto. de Geociências da UEL. E-mail: defranca@uel.br.

4 Profa. Dra. do Depto. de Ciências Biológicas da UEL. E-mail: aovieira@uel.br. Departamento de Agronomia, Universidade Estadual de Londrina, Campus Universitário CCA - Londrina - PR - 86051-970 - (43) 33714555.
} 
TRABAQUINI, K.; et al. Análise espacial de fragmentos florestais...

\section{INTRODUÇÃO}

As espécies arbóreas são de grande importância para a fauna, pela quantidade de recursos que oferece, como flores, frutos e néctar, e também pela importância econômica e cultural, servindo de alimento, medicamento, combustível e vestuário para um número considerável de pessoas no mundo todo (UNEP, 2006).

A espécie Jacaratia spinosa (Aubl.) A.DC. é uma espécie nativa arbórea de ampla distribuição geográfica (CORREIA, 1984), pertencente ao grupo das Angiospermas, e à família Caricaceae (BAKER, 1976). O gênero Jacaratia A.DC. contém cerca de dez espécies tropicais e subtropicais, das quais seis são exclusivas da América. No Brasil, são encontradas três espécies J. corumbensis Kuntze, J. heptaphylla (Vell.) A.DC. e J. spinosa (Aubl.) A.DC. (LIMA \& PIRANI, 2002).

No Brasil, ocorre no Amapá, Pará, Bahia, Espírito Santo, Minas Gerais, Mato Grosso do Sul e do Rio de Janeiro até o Rio Grande do Sul. É conhecida pelos nomes populares de jacarati jaraiatiá, mamão-do-mato e mamãozinho-do-mato (LIMA; PIRANI, 2002). É uma planta lactescente, dióica e de caule espinhento, de 10-20 m de altura, com tronco de $70-90 \mathrm{~cm}$ de diâmetro, com folhas compostas palmatilobadas, com 8-12 folíolos glabros (LORENZI, 2002). Segundo Moraes et al. (2005), esta espécie pode ser classificada como sendo pioneira antrópica, ou seja, estão associadas a extremos períodos de exposição à luz e possuem crescimento rápido.

Ocorre sempre em baixa densidade, contudo, deve ser presença obrigatória em qualquer reflorestamento heterogêneo destinado à recomposição da vegetação de áreas degradadas de preservação permanente, pois, como planta pioneira, é adaptada à luminosidade direta e de rápido crescimento. (LORENZI, 2002)

Esta espécie possui um grande potencial de exploração, pois a partir do caule e dos frutos desta árvore podem ser fabricados doces e compotas e ainda apresenta outras finalidades industriais, o que a torna uma fonte de renda para os agricultores, também sendo indicada para a recomposição de áreas degradadas e planos de manejo florestal. Trabalhos realizados por Muniz et al. (2004) mostram que as fibras produzidas pela planta podem originar celulose e papel de ótima qualidade, alta resistência e baixo custo.

Além da importância da espécie, ela está incluída na Lista Vermelha de plantas ameaçadas de extinção no estado do Paraná (PARANÁ, 1995) e também no estado do Rio Grande do Sul (SEMA, 2002). É nesse contexto que a vegetação, em especial as formações florestais, merece destaque como um dos mais importantes indi- cadores das condições ambientais, exercendo um papel essencial no equilíbrio dinâmico. O processo de extinção de qualquer espécie afeta diretamente o ecossistema, causando graves conflitos socioambientais.

Ao final do século XIX, as florestas nativas do estado do Paraná originalmente cobriam mais de $80 \%$ do seu território, mas durante o século passado foram reduzidas progressivamente. Entre as diferentes regiões fitoecológicas paranaenses, aquela sob o domínio da Floresta Estacional Semidecidual foi a mais antropizada, correspondendo atualmente a menos de $4 \%$ de sua superfície original. (RODERJAN et al. 2002)

Existem dois tipos de fragmentos florestais: remanescentes florestais e manchas de floresta. Os remanescentes florestais são aqueles fragmentos originados por desmatamento a partir de uma floresta contínua, como é o caso no norte do estado do Paraná. As manchas de floresta são naturais e aparecem em regiões de campo aberto. Na bacia do rio Tibagi, onde a área de estudo está localizada, existem os dois tipos de fragmentos florestais: remanescentes florestais e manchas de floresta. (ANJOS; BOÇON, 1999)

Estudos de análise de fragmentos florestais por meio de sensoriamento remoto são feitos principalmente com o objetivo de se obter informações que sustentem possíveis planos de conservação, como nos trabalhos de Ditt (2002) e Metzger (2003), ambos desenvolvidos em áreas onde a cobertura vegetal nativa foi aos poucos sendo substituída por pequenos remanescentes florestais.

Os fatores que influenciam a estrutura e a dinâmica das comunidades vegetais manifestam-se em várias escalas. Estas podem ser locais, como características de solos, alagamentos e drenagem (KALLIOLA et al., 1988); regionais, como as médias de temperatura e a ocorrência de geadas e quantidade e distribuição de chuvas (VELOSO et al., 1991); ou continentais, como as variações climáticas de larga escala (WALTER, 1986). Pois os fatores do meio interferem e atuam em conjunto, com interações e relações complexas na distribuição e no crescimento das espécies vegetais.

O presente estudo teve por finalidade analisar o contexto espacial dos fragmentos onde houve o registro da espécie Jacaratia spinosa (Aubl.) A.DC, através de técnicas de sensoriamento remoto e por conseqüência fazer uma avaliação da estrutura da espécie. Os resultados do estudo reuniram informações importantes que substanciaram as cartas temáticas na dimensão ambiental sobre a distribuição e a localização da espécie no norte do estado do Paraná. 


\section{MATERIAIS E MÉTODO}

\section{ÁREA DE ESTUDOS}

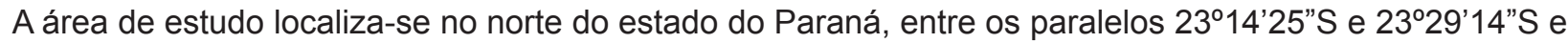
meridianos 5057'15"W e 51 ${ }^{\circ} 23^{\prime} 14$ "W, no Terceiro Planalto Paranaense, contemplando os municípios de Londrina, Ibiporã, Cambé e Arapongas. (Tabela 1 e Figura 1)

TABELA 1 - MUNICÍPIOS E A LOCALIZAÇÃO DOS FRAGMENTOS FLORESTAIS NO PARANÁ

\begin{tabular}{ccc}
\hline \multirow{2}{*}{ MUNICÍPIOS } & $\begin{array}{c}\text { FRAGMENTOS FLORESTAIS VISITA- } \\
\text { DOS }\end{array}$ & LOCALIZAÇÃO \\
\hline \multirow{2}{*}{ Ibiporã } & 1 & Horto Municipal de Ibiporã \\
\cline { 2 - 3 } & 2 & Fazenda Doralice \\
\hline Cambé & 3 & Parque Peroba Rosa \\
\hline \multirow{2}{*}{ Londrina } & 4 & Parque Municipal Arthur Thomas \\
\cline { 2 - 3 } & 6 & Parque Estadual Mata dos Godoy \\
\cline { 2 - 3 } & 7 & Fazenda Santa Helena \\
\hline Arapongas & 5 & Fazenda Solana \\
\hline
\end{tabular}

A vegetação originalmente dominante era a Floresta Estacional Semidecídua, que sofreu um processo intenso de fragmentação a partir do século XIX, resultando numa paisagem composta por uma miríade de pequenos fragmentos, somando apenas 2 a $4 \%$ da área antes ocupada por florestas. (IPARDES, 1993)

O clima da região é classificado conforme mé- todo Köeppen como Cfa, ou seja, clima subtropical, com temperatura média no mês mais frio inferior a $18^{\circ} \mathrm{C}$ (mesotérmico), média anual de precipitação de 1.600 $\mathrm{mm}$, temperatura média no mês mais quente acima de $22^{\circ} \mathrm{C}$, com verões quentes, geadas pouco freqüentes e tendência de concentração das chuvas nos meses de verão, contudo sem estação seca definida. (IAPAR, 1994)

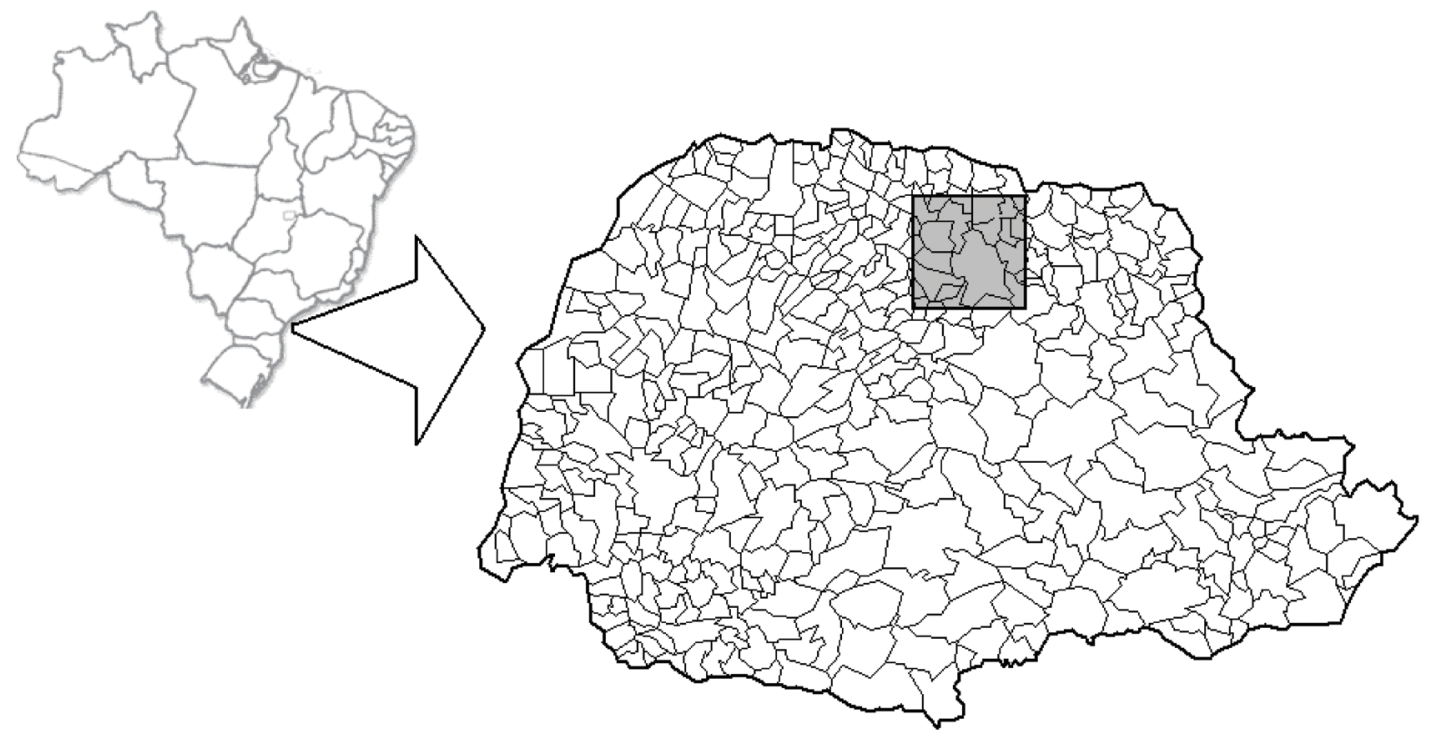


A figura 2 representa a área de estudo e a localização dos fragmentos florestais numerados de 1 a 7 , onde foram coletados os dados para o desenvolvimento do trabalho.

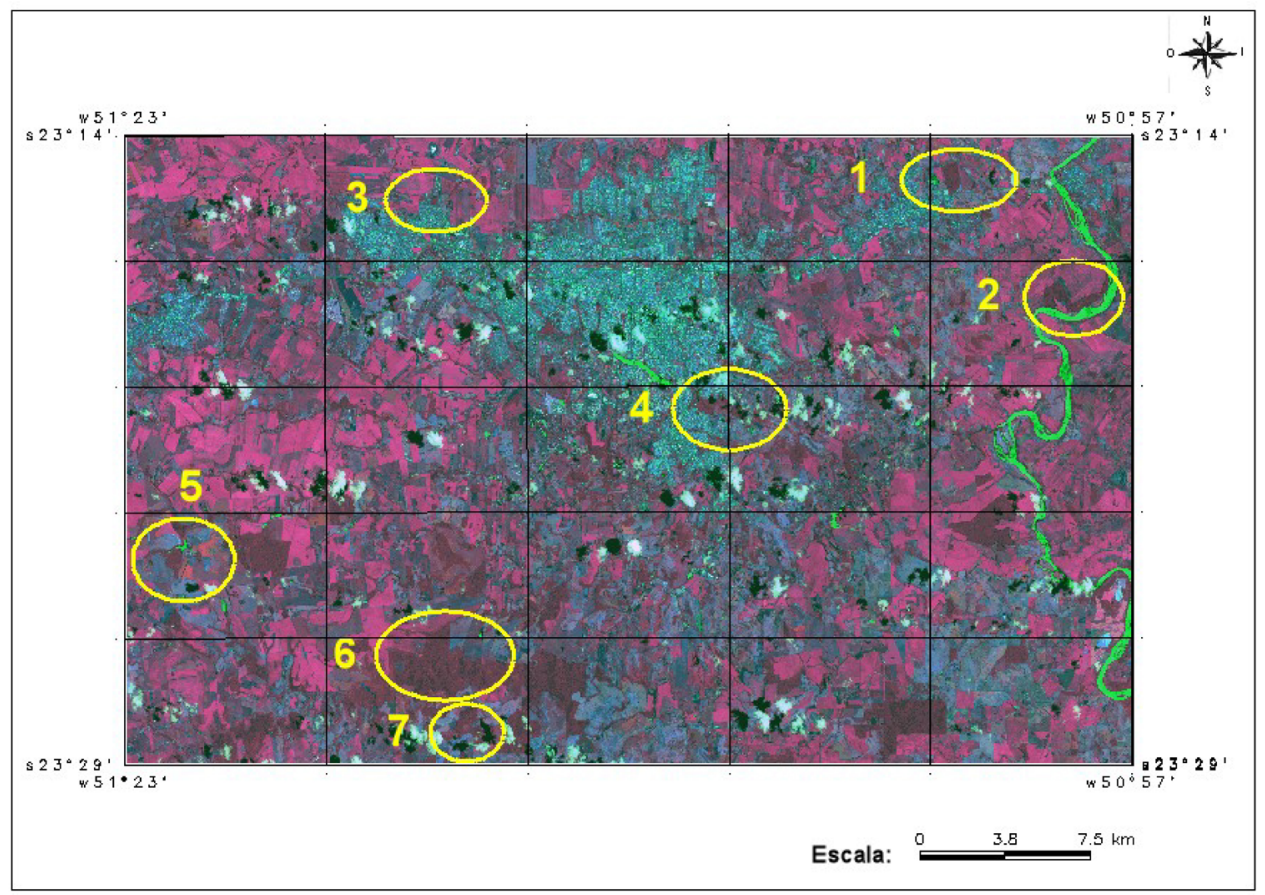

FIGURA 2 - ÁREA DE ESTUDO - LOCALIZAÇÃO DOS FRAGMENTOS FLORESTAIS NO NORTE DO PARANÁ - COMPOSIÇÃO COLORIDA RGB NA SEQÜËNCIA 1, 2, 3 - EXTRAÇÃO DA CENA SPOT 5 HRG; BASE: 707_396; PASSAGEM DATA:18/1/2004 TRATAMENTO: TRABAQUINI (2007)

\section{MATERIAIS}

O levantamento de dados foi realizado utilizandose um receptor GPS (Global Positioning System) de navegação ETREX, modelo Vista, com o qual as plantas da espécie $J$. spinosa foram localizadas na paisagem. No local foram registradas as coordenadas geográficas (latitude e longitude) e altitude. Foram registrados parâmetros como altura da planta e CAP (Circunferência à Altura do Peito) dos indivíduos.

Foi utilizado o software SPRING (Sistema para Processamento de Informações Georreferenciadas) / INPE, versão 4.3.3, para o tratamento digital das imagens orbitais, para localizar e estimar os pontos de localização e distribuição da espécie a partir dos dados de localização dos indivíduos amostrados.

Realizou-se a caracterização no contexto ambiental da área de estudo, através de aplicação do Sensoriamento Remoto Orbital, utilizado como apoio tecnológico o SIG, especificamente um dos módulos do software, SPRING/INPE. A imagem utilizada foi do satélite SPOT 5 (Satellite pour l'Observation de la Terre), com data de passagem 18/1/2004, órbita-ponto 707_396; sensor HRG (High-Resolution Geometric); uma faixa imageada de $60 \mathrm{~km}$; resolução de $10 \mathrm{~m}$ nas bandas multiespectral 1, 2 e 3 , e resolução de $2,5 \mathrm{~m}$ na banda pancromática.

\section{MÉTODO}

Elaborou-se um banco de dados georreferenciado contendo como informação cadastral as coordenadas geográficas coletadas dos indivíduos, realizado em trabalho de campo.

No trabalho de escritório, foram utilizadas as imagens do satélite SPOT 5, bandas B1, B2 e B3, para o processamento digital das imagens. Foram aplicadas técnicas de pré-tratamento digital nas imagens, a fim de corrigir distorções geométricas e correções radiométricas para a maior confiabilidade à classificação e interpretação das mesmas. 
Para facilitar os estudos e reduzir o tempo de processamento computacional das imagens, cada área de estudo foi selecionada no módulo IMPIMA 4.3.3 e importada no SPRING 4.3.3 no formato de arquivo "grib". Posteriormente, para cada fragmento foi feito um recorte da área de interesse na escala 1/10.000, adquirindo também as informações que estão no entorno dos fragmentos, tendo como exceção o fragmento n. 6, que foi conduzido na escala $1 / 30.000$ devido a sua maior dimensão, como constatado na Figura 2.

Para cada fragmento foi realizado o georreferenciamento com o auxílio de cinco pontos de controle registrados com GPS nas campanhas de campo. As bandas passaram por um ajuste de contraste com o objetivo de melhorar a qualidade visual da imagem e pelo método de Análise dos Componentes Principais (ACP), gerando um novo conjunto de imagens com valores de reflectâncias mais confiáveis. Após isso, foi composta uma imagem em Composição Colorida RGB, com as bandas na seqüência B1, B2, B3, para auxiliar na interpretação e classificação da imagem.
O principal objetivo da aplicação da ACP foi sintetizar o conteúdo dos dados originais, eliminando as informações redundantes que existiam entre as diferentes bandas do sensor, reduzindo assim a dimensão original das variáveis para um conjunto menor de variáveis, mas contendo toda a informação original. (MENEZES; NETTO, 2001)

A próxima etapa foi a segmentação da banda 1, que teve por objetivo fragmentar a imagem em unidades homogêneas, considerando algumas de suas características, como o nível de cinza dos pixels, textura e contraste da imagem (MOREIRA, 2005). O algoritmo de Segmentação por Crescimento de Regiões, disponível no SPRING, depende da definição das duas variáveis, grau de similaridade e a área mínima em pixels para o estabelecimento de uma região, nas quais para cada área de estudo foram definidas variáveis distintas, representadas na Tabela 2.

TABELA 2 - VALORES DE SIMILARIDADE E ÁREA UTILIZADOS NA SEGMENTAÇÃO DAS ÁREAS DE ESTUDO - SPOT 5 - BANDA 1

\begin{tabular}{cccccccc}
\hline FRAGMENTOS & 1 & 2 & 3 & 4 & 5 & 6 & 7 \\
\hline Similaridade & 18 & 14 & 11 & 17 & 18 & 15 \\
\hline Área (pixels) & 30 & 15 & 15 & 25 & 22 & 15 & 28 \\
\hline
\end{tabular}

Com a interpretação da imagem em Composição Colorida, selecionaram-se os temas para a classificação da imagem da área de estudos. Para a classificação foi utilizado o classificador de Bhattacharya. Esse método, partindo da imagem segmentada, envolve uma fase de aquisição e análise de amostras fornecidas, com o objetivo de aperfeiçoar o mapeamento final. Através dessa etapa foram realizadas a interpretação e a identificação da imagem dos aspectos dos usos dos solos, determinados em sete classes: agricultura, solo exposto, mata primária, mata ciliar, urbano, água e pastagem.

A última etapa foi a pós-classificação, através da qual as variáveis Peso e Limiar, ajustadas pelo usuário, são aplicadas à imagem já classificada, com o objetivo de eliminar os pixels que ficaram isolados, ou seja, uniformizar os temas.

Com a edição das imagens concluídas e a área já caracterizada, foi utilizado o banco de dados dos indivíduos com a posição geográfica coletadas a campo, assim sendo locadas na imagem. Com isso, elaboraramse as sete cartas temáticas, indicando a distribuição da espécie $J$. spinosa, a localização e as classes que cada Fragmento Florestal está inserido quanto ao contexto ambiental da região.

Para a avaliação da população de J. spinosa amostrada, foram calculadas, segundo Felfili \& Rezende (2003), a Densidade Absoluta (DA), que é obtida pela contagem do número de indivíduos amostrados de uma determinada espécie na área amostral em hectare, e a Dominância Absoluta (DoA), que expressa a proporção de tamanho ou de cobertura da espécie em estudo. Abaixo são apresentadas as fórmulas utilizadas para os cálculos.

\section{$\mathrm{DA}=\mathrm{n} /$ área}

Onde:

$\mathrm{n}=\mathrm{n}$. total de indivíduos amostrados na área;

área $=$ área total em hectare.

\section{DoA = gi / área}

Onde:

gi $=\pi / 4^{*} d^{2}$

$\mathrm{d}=\mathrm{DAP}$ (diâmetro à altura do peito) de cada indivíduo, em metros. 


\section{RESULTADOS E DISCUSSÃO}

As diferenças entre as condições de usos dos solos e as características da paisagem no entorno dos fragmentos envolveram as sete classes, no contexto ambiental dos remanescentes onde se localizam os indivíduos J. spinosa.
As características de cada fragmento são demonstradas na Tabela 3, onde a área do fragmento e as classes de uso do solo podem ser observados. Do total, quatro Classes são sistemas fisionômicos resultantes de ações antrópicas (agricultura, solo exposto, urbano e pastagem) e três são sistemas naturais (mata primária, mata ciliar e água).

TABELA 3 - CARACTERÍSTICAS E PERCENTAGENS DE CADA FRAGMENTO FLORESTAL E SUAS RESPECTIVAS CLASSES DE USO DO SOLO

\begin{tabular}{|c|c|c|c|c|c|c|c|c|}
\hline $\begin{array}{l}\text { N. E NOME DOS } \\
\text { FRAGMENTOS }\end{array}$ & $\begin{array}{c}\text { ÁREA DO } \\
\text { FRAGMENTO } \\
\text { (ha) }\end{array}$ & $\begin{array}{c}\text { AGRICULTURA } \\
(\%)\end{array}$ & $\begin{array}{c}\text { SOLO } \\
\text { EXPOSTO } \\
(\%) \\
\end{array}$ & $\begin{array}{c}\text { MATA } \\
\text { PRIMÁRIA } \\
(\%) \\
\end{array}$ & $\begin{array}{c}\text { MATA } \\
\text { CILIAR } \\
(\%) \\
\end{array}$ & $\begin{array}{l}\text { URBANO } \\
\text { (\%) }\end{array}$ & $\begin{array}{c}\text { ÁGUA } \\
(\%)\end{array}$ & $\begin{array}{c}\text { PASTAGEM } \\
\text { (\%) }\end{array}$ \\
\hline $\begin{array}{l}\text { 1. Horto Municipal de } \\
\text { Ibiporã }\end{array}$ & 71,44 & 20,35 & 50,91 & 13,64 & 0,00 & 15,08 & 0,00 & 0,00 \\
\hline 2. Fazenda Doralice & 92,11 & 38,04 & 11,81 & 29,00 & 0,00 & 0,00 & 21,15 & 0,00 \\
\hline 3. Parque Peroba Rosa & 9,13 & 40,21 & 29,61 & 1,88 & 5,19 & 23,07 & 0,00 & 0,00 \\
\hline $\begin{array}{l}\text { 4. Parque Municipal } \\
\text { Arthur Thomas }\end{array}$ & 84,58 & 0,00 & 21,83 & 24,61 & 0,00 & 38,92 & 0,68 & 13,94 \\
\hline 5. Fazenda Solana & 58,72 & 30,56 & 42,13 & 17,49 & 0,00 & 0,00 & 1,77 & 8,03 \\
\hline $\begin{array}{l}\text { 6. Parque Estadual } \\
\text { Mata dos Godoy }\end{array}$ & 650 & 23,05 & 21,86 & 55,08 & 0,00 & 0,00 & 0,00 & 0,00 \\
\hline $\begin{array}{l}\text { 7. Fazenda Santa } \\
\text { Helena }\end{array}$ & 53,63 & 27,69 & 23,31 & 48,43 & 0,00 & 0,00 & 0,00 & 0,55 \\
\hline
\end{tabular}

Nos estudos, notou-se que o Fragmento n. 1, situado no Horto Florestal de Ibiporã, possui altitude média de $424 \mathrm{~m}$ e apresenta quatro indivíduos com CAP médio de $204 \mathrm{~cm}$, evidenciando árvores de grande porte, antigas e preservadas. Quanto ao uso do solo no entorno do fragmento, a área apresentou o maior índice de ocupação da Classe Solo Exposto, com 50,91\%.

O Fragmento n. 2, localizado no município de Ibiporã, à margem esquerda do Rio Tibagi, possui oito indivíduos com CAP médio de $134 \mathrm{~cm}$ e a menor altitude da área de estudo, com $342 \mathrm{~m}$, demonstrando que a altitude é crescente de leste para oeste na área de estudo. Quanto ao uso do solo do entorno do fragmento, a área apresentou o segundo maior índice de ocupação da Classe Agricultura com 38,04\%.

O Fragmento n. 3, situado no município de Cambé, está localizado numa área de transição da área urbana para a área rural, representando assim o maior índice da Classe Agricultura, com 40,21\%. Essa área apresenta altitude média de $597 \mathrm{~m}$ e apenas três indivíduos com CAP médio de $110 \mathrm{~cm}$.
O Fragmento n. 4 está situado na área urbana do município de Londrina, conseqüentemente possuindo o maior índice da Classe Urbano, com $38,92 \%$, e com altitude média de $498 \mathrm{~m}$. Apesar do alto índice de urbanização no entorno do fragmento, este possui o maior número de indivíduos registrado, com doze árvores e CAP médio de $137 \mathrm{~cm}$, podendo ser explicado pelo fato de que este fragmento é um Parque Municipal de Conservação e, com isto, as atividades da área são fiscalizadas.

O Fragmento n. 5, situado no município de Arapongas, está localizado na área rural deste, sendo o seu entorno ocupado principalmente pela Classe Solo Exposto, com 42,13\%, e com a maior média de altitude, com $694 \mathrm{~m}$. O porte dos nove indivíduos registrados foram os menores da área de estudo, com CAP médio de $68 \mathrm{~cm}$.

O Fragmento n. 6, está situado na área rural do município de Londrina, e também é uma Unidade de Conservação como o Fragmento n. 4, apesar de apresentar o maior índice da Classe Mata Primária, com $55,08 \%$ e altitude média de $611 \mathrm{~m}$, só foram encontrados quatro indivíduos com CAP médio de $81 \mathrm{~cm}$. 
O Fragmento n. 7, situado na área rural do município de Londrina, apresentou no seu entorno o segundo maior índice da Classe Mata Primária, com 48,43\% e altitude média de $577 \mathrm{~m}$. Foram registrados no local cinco indivíduos com CAP médio de $113 \mathrm{~cm}$.
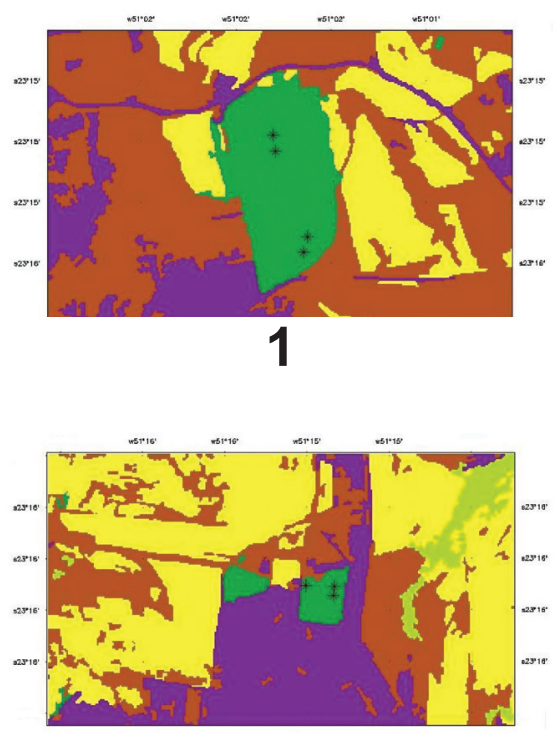

3

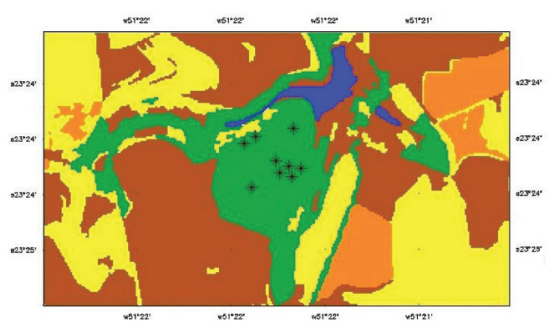

5

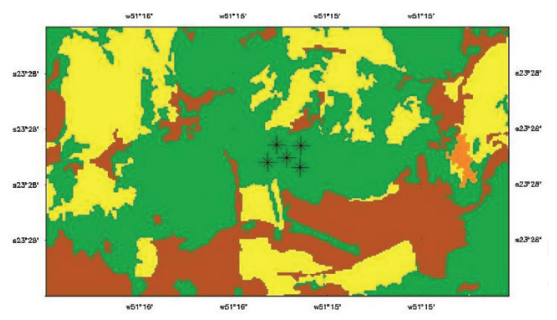

7
A Figura 3 a seguir apresenta a distribuição da espécie e as classes de uso do solo pertencentes a cada fragmento de estudo.
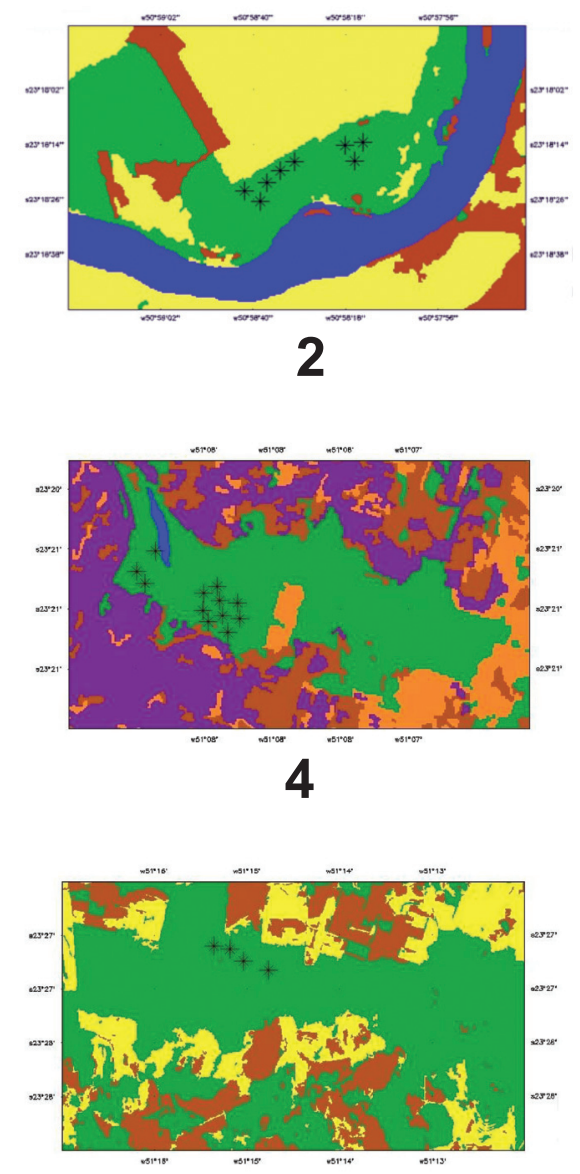

6

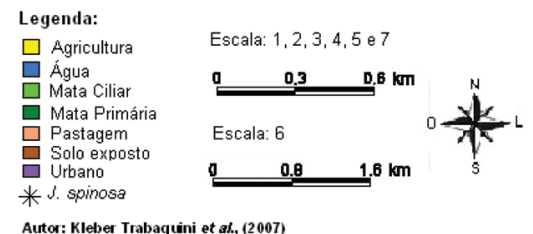

FIGURA 3 - MAPAS TEMÁTICOS DE USO DO SOLO DOS FRAGMENTOS E OCORRÊNCIA DE JACARATIA SPINOSA (AUBL.) A.DC. 1: HORTO MUNICIPAL DE IBIPORÃ. 2: FAZENDA DORALICE. 3: PARQUE PEROBA ROSA. 4: PARQUE MUNICIPAL ARTHUR THOMAS. 5: FAZENDA SOLANA. 6: PARQUE ESTADUAL MATA DOS GODOY. 7: FAZENDA SANTA HELENA.

Em relação às características biométricas dos indivíduos que foram amostrados a campo, os dados são apresentados na Tabela 4, onde se pode observar a altura, o CAP e a altitude onde cada indivíduo foi registrado, 
TRABAQUINI, K.; et al. Análise espacial de fragmentos florestais...

e oriundos das campanhas de campo realizadas durante os meses de junho a setembro de 2007. TABELA 4 - CARACTERÍSTICAS BIOMÉTRICAS E DE ALTITUDE DE JACARATIA SPINOSA (AUBL.) A.DC.

\begin{tabular}{|c|c|c|c|}
\hline FRAGMENTOS & ALTURA DA PLANTA & CAP* & ALTITUDE \\
\hline \multirow{4}{*}{ 1. Horto Municipal de Ibiporã } & $14 \mathrm{~m}$ & $228 \mathrm{~cm}$ & $444 \mathrm{~m}$ \\
\hline & $12 \mathrm{~m}$ & $194 \mathrm{~cm}$ & $421 \mathrm{~m}$ \\
\hline & $14 \mathrm{~m}$ & $200 \mathrm{~cm}$ & $402 \mathrm{~m}$ \\
\hline & $16 \mathrm{~m}$ & $197 \mathrm{~cm}$ & $430 \mathrm{~m}$ \\
\hline \multirow{8}{*}{ 2. Fazenda Doralice } & $11 \mathrm{~m}$ & $137 \mathrm{~cm}$ & $361 \mathrm{~m}$ \\
\hline & $15 \mathrm{~m}$ & $170 \mathrm{~cm}$ & $361 \mathrm{~m}$ \\
\hline & $7 \mathrm{~m}$ & $144 \mathrm{~cm}$ & $361 \mathrm{~m}$ \\
\hline & $11 \mathrm{~m}$ & $144 \mathrm{~cm}$ & $342 \mathrm{~m}$ \\
\hline & $15 \mathrm{~m}$ & $103 \mathrm{~cm}$ & $373 \mathrm{~m}$ \\
\hline & $7 \mathrm{~m}$ & $77 \mathrm{~cm}$ & $371 \mathrm{~m}$ \\
\hline & $13 \mathrm{~m}$ & $122 \mathrm{~cm}$ & $362 \mathrm{~m}$ \\
\hline & $15 \mathrm{~m}$ & $183 \mathrm{~cm}$ & $360 \mathrm{~m}$ \\
\hline \multirow{3}{*}{ 3. Parque Peroba Rosa } & $10 \mathrm{~m}$ & $44 \mathrm{~cm}$ & $600 \mathrm{~m}$ \\
\hline & $11 \mathrm{~m}$ & $160 \mathrm{~cm}$ & $598 \mathrm{~m}$ \\
\hline & $16 \mathrm{~m}$ & $126 \mathrm{~cm}$ & $593 \mathrm{~m}$ \\
\hline \multirow{12}{*}{ 4. Parque Municipal Arthur Thomas } & $7 \mathrm{~m}$ & $133 \mathrm{~cm}$ & $526 \mathrm{~m}$ \\
\hline & $12 \mathrm{~m}$ & $170 \mathrm{~cm}$ & $526 \mathrm{~m}$ \\
\hline & $16 \mathrm{~m}$ & $191 \mathrm{~cm}$ & $501 \mathrm{~m}$ \\
\hline & $14 \mathrm{~m}$ & $202 \mathrm{~cm}$ & $503 \mathrm{~m}$ \\
\hline & $9 \mathrm{~m}$ & $53 \mathrm{~cm}$ & $503 \mathrm{~m}$ \\
\hline & $7 \mathrm{~m}$ & $90 \mathrm{~cm}$ & $503 \mathrm{~m}$ \\
\hline & $13 \mathrm{~m}$ & $170 \mathrm{~cm}$ & $494 \mathrm{~m}$ \\
\hline & $16 \mathrm{~m}$ & $185 \mathrm{~cm}$ & $506 \mathrm{~m}$ \\
\hline & $13 \mathrm{~m}$ & $128 \mathrm{~cm}$ & $483 \mathrm{~m}$ \\
\hline & $9 \mathrm{~m}$ & $47 \mathrm{~cm}$ & $469 \mathrm{~m}$ \\
\hline & $16 \mathrm{~m}$ & $134 \mathrm{~cm}$ & $459 \mathrm{~m}$ \\
\hline & $12 \mathrm{~m}$ & $152 \mathrm{~cm}$ & $505 \mathrm{~m}$ \\
\hline \multirow{9}{*}{ 5. Fazenda Solana } & $12 \mathrm{~m}$ & $103 \mathrm{~cm}$ & $682 \mathrm{~m}$ \\
\hline & $9 \mathrm{~m}$ & $48 \mathrm{~cm}$ & $702 \mathrm{~m}$ \\
\hline & $11 \mathrm{~m}$ & $74 \mathrm{~cm}$ & $702 \mathrm{~m}$ \\
\hline & $6 \mathrm{~m}$ & $26 \mathrm{~cm}$ & $702 \mathrm{~m}$ \\
\hline & $10 \mathrm{~m}$ & $49 \mathrm{~cm}$ & $702 \mathrm{~m}$ \\
\hline & $15 \mathrm{~m}$ & $120 \mathrm{~cm}$ & $703 \mathrm{~m}$ \\
\hline & $7 \mathrm{~m}$ & $44 \mathrm{~cm}$ & $691 \mathrm{~m}$ \\
\hline & $14 \mathrm{~m}$ & $121 \mathrm{~cm}$ & $686 \mathrm{~m}$ \\
\hline & $6 \mathrm{~m}$ & $32 \mathrm{~cm}$ & $683 \mathrm{~m}$ \\
\hline \multirow{4}{*}{ 6. Parque Estadual Mata dos Godoy } & $12 \mathrm{~m}$ & $126 \mathrm{~cm}$ & $608 \mathrm{~m}$ \\
\hline & $8 \mathrm{~m}$ & $94 \mathrm{~cm}$ & $612 \mathrm{~m}$ \\
\hline & $11 \mathrm{~m}$ & $84 \mathrm{~cm}$ & $639 \mathrm{~m}$ \\
\hline & $4 \mathrm{~m}$ & $20 \mathrm{~cm}$ & $586 \mathrm{~m}$ \\
\hline \multirow{5}{*}{ 7. Fazenda Santa Helena } & $18 \mathrm{~m}$ & $143 \mathrm{~cm}$ & $577 \mathrm{~m}$ \\
\hline & $12 \mathrm{~m}$ & $123 \mathrm{~cm}$ & $575 \mathrm{~m}$ \\
\hline & $12 \mathrm{~m}$ & $77 \mathrm{~cm}$ & $575 \mathrm{~m}$ \\
\hline & $10 \mathrm{~m}$ & $90 \mathrm{~cm}$ & $575 \mathrm{~m}$ \\
\hline & $13 \mathrm{~m}$ & $132 \mathrm{~cm}$ & $583 \mathrm{~m}$ \\
\hline
\end{tabular}


${ }^{*}$ CAP $=$ CIRCUNFERÊNCIA À ALTURA DO PEITO.

A partir dos dados das Tabelas 3 e 4, pode-se fazer uma avaliação momentânea da estrutura da espécie. Foram calculados a Densidade absoluta, que representa o número de indivíduos de uma determina espécie na área, e a Dominância absoluta, que expressa área basal de uma espécie na área, sendo os resultados apresentados na Tabela 5.

TABELA 5 - DENSIDADE ABSOLUTA (DA) E DOMINÂNCIAABSOLUTA (DOA) DOS INDIVÍDUOS ENCONTRADOS NOS FRAGMENTOS

\begin{tabular}{lccc}
\hline \multicolumn{1}{c}{ FRAGMENTOS FLORESTAIS } & N. DE INDIVÍDUOS & DA (ind.ha') & \left.${\text { DoA }\left(\mathbf{m}^{\mathbf{2}} \text {. ha }\right.}^{\mathbf{1}}\right)$ \\
\hline 1. Horto Municipal de Ibiporã & 4 & 0,05 & 0,18 \\
2. Fazenda Doralice & 8 & 0,08 & 0,13 \\
3. Parque Peroba Rosa & 3 & 0,32 & 0,37 \\
4. Parque Municipal Arthur Thomas & 12 & 0,14 & 0,23 \\
5. Fazenda Solana & 9 & 0,15 & 0,04 \\
6. Parque Estadual Mata dos Godoy & 4 & 0,006 & 0,003
\end{tabular}

7. Fazenda Santa Helena

5

0,09

0,09

Os resultados da Densidade Absoluta (DA) e da Dominância Absoluta (DoA) são valores muito baixos quando comparados com estudos fitossociológicos nos fragmentos estudados: no Parque Estadual Mata dos Godoy a DA foi calculada em 1,67 e a DoA em 0,17 (SILVEIRA, 1993); já na Fazenda Solana, a DA foi de 5 e a DoA de 0,014 (CERVIGNE, 2007). Outros estudos conduzidos em outras áreas, como Battilani et al. (2005), estudando um trecho da mata ciliar do Rio da Prata, Jardim, MS, encontraram 4,44 (DA) e 0,79 (DoA), porém com a área de estudo sendo de 0,9 ha. Nos estudos de Silva e Soares (2001), num fragmento de 112 ha, situado no município de São Carlos, sob domínio da floresta estacional semidecídua, os índices foram de 21 (DA) e 0,71 (DoA), novamente demonstrando ser índices altos, quando comparados com este estudo. Apesar dos resultados baixos da DAe DoA, a taxa de sobrevivência da espécie é alta, com 95,8\%, como podemos constatar nos estudos de Moraes et al. (2005).

Em estudo desenvolvido por Santos (2007), com dieta e dispersão pelo bugio-ruivo, Alouatta clamitans, em um dos fragmentos estudados (Fazenda Doralice), foi constatado que os frutos da espécie Jacaratia spinosa foram um dos principais alimentos consumidos por esse primata. Após as sementes passarem pelo trato digestivo desse animal, a taxa de germinação foi de 68\%, e a distância média de dispersão dos frutos promovida por esses animais foi de 79,6 m. Esses dados podem ser relacionados aos resultados obtidos, pois foi verificada a baixa densidade dessa espécie e em pequenos agrupamentos no interior dos fragmentos, o que pode ser constatado nos mapas temáticos (figura 3).

A distribuição da freqüência de classes de circunferência (CAP) dos indivíduos registrados mostra que a maior concentração de indivíduos ocorreu na classe 110 a $140 \mathrm{~cm}$, o que representa 24,44\% dos indivíduos (figura 4), porém o menor CAP registrado foi de $26 \mathrm{~cm}$ e o maior de $228 \mathrm{~cm}$. Em um estudo que acompanhou o desenvolvimento do jacaratiá por quatro anos, foi visto que a média do diâmetro ao nível do solo foi de $40 \mathrm{~cm}$ (MORAES et al. 2005), enquanto que Battilani et al. (2005), em uma localidade mineira, registraram como o maior CAP $234 \mathrm{~cm}$.

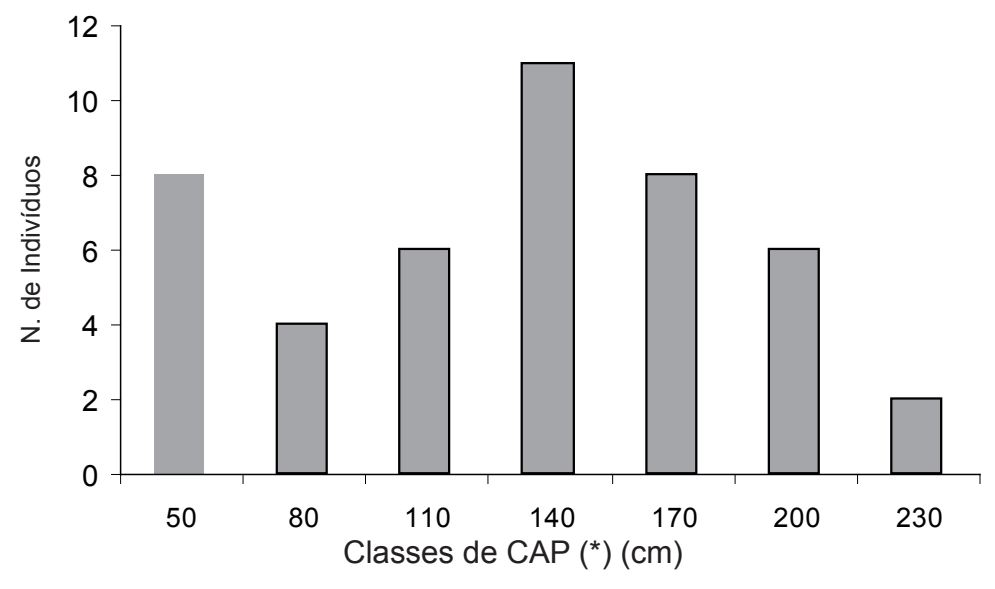

* CAP = CIRCUNFERÊNCIA À ALTURA DO PEITO 
TRABAQUINI, K.; et al. Análise espacial de fragmentos florestais...

FIGURA 4 - DISTRIBUIÇÃO DA FREQÜÊNCIA DE CLASSES DE CAP (CM) DE JACARATIA SPINOSA (AUBL.) A.DC.

A Figura 5 apresenta dados de distribuição de freqüência de classes de altura dos indivíduos amostrados. Verifica-se que $26,66 \%$ dos indivíduos estão no intervalo de 9 a $11 \mathrm{~m}$ de altura, contudo o menor indivíduo encontrado foi de $6 \mathrm{~m}$ e o maior com $18 \mathrm{~m}$ de altura. Nos estudos de Lima e Pirani (2002), os autores citam para essa espécie indivíduos de 6 a $25 \mathrm{~m}$ de altura.

FIGURA 5 - DISTRIBUIÇÃO DA FREQÜÊNCIA DE CLASSES DE ALTURA DE JACARATIA SPINOSA (AUBL.) A.DC.

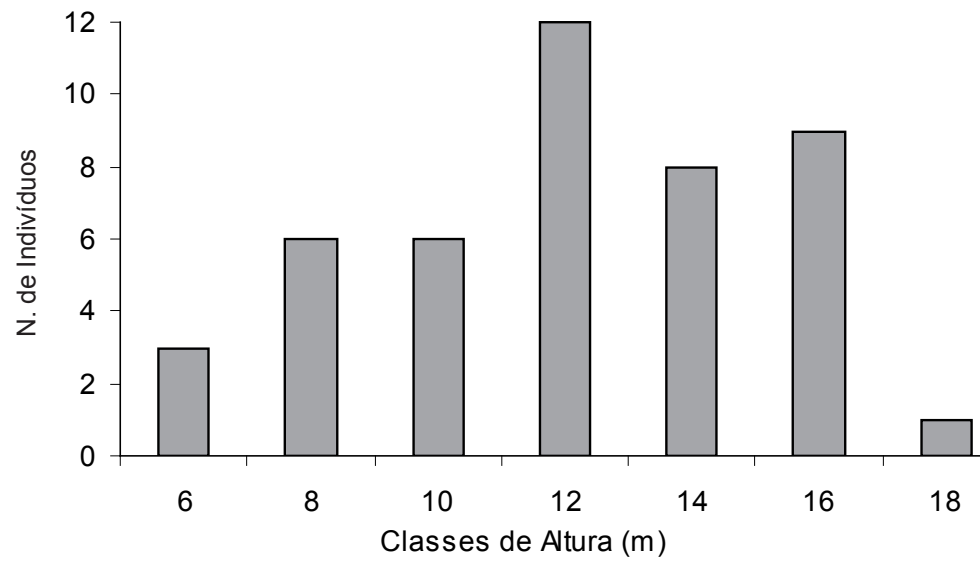

Analisando a Tabela 4, podemos ainda destacar a variação de altitude registrada para cada indivíduo localizado na área de estudo, pois nessa área ocorre um cenário geográfico com variações de altitude de 342 a $703 \mathrm{~m}$ acima do nível do mar.

\section{CONSIDERAÇÕES FINAIS}

A avaliação da paisagem com a utilização de imagem do satélite SPOT 5, aliada às técnicas de sensoriamento remoto orbital, demonstram que esta pode ser uma "ferramenta" útil para o monitoramento da distribuição da espécie J. spinosa e a discriminação das classes de uso do solo que estão inseridas no contexto ambiental.

Evidencia que a espécie $J$. spinosa está inserida em fragmentos florestais onde as atividades referentes às classes de uso do solo colocam os fragmentos sujeitos à ação de alguns tipos de perturbações, como uso de agroquímicos e queimadas na agricultura. Nas áreas vizinhas, a Classe Urbano, há deposição principalmente de resíduos sólidos urbanos e da construção civil no entorno e até no interior da mata. E também áreas de solos expostos, quando prolongadas por muito tempo, podendo vir a causar erosão e assoreamento dos corpos d'água.

As condições de perturbações naturais e antrópicas podem estar contribuindo para a baixa abundância de indivíduos nos fragmentos amostrados, indicando uma alta susceptibilidade à extinção local da espécie, 
TRABAQUINI, K.; et al. Análise espacial de fragmentos florestais...

caso ocorra morte ou corte desses indivíduos amostrados. Outra importante questão a ser abordada é que os indivíduos estão distribuídos em pequenos grupos, com árvores de CAP elevado, não ocorrendo um número significativo de indivíduos jovens nos fragmentos. Tendo em vista que as taxas de germinação e sobrevivência dessa espécie são relativamente altas, este fato pode ser justificado pela diminuição da fauna nas matas, ou seja, os animais que são responsáveis pela dispersão das sementes podem estar em número reduzido, já que essa espécie é dependente da distribuição zoocórica.

Esta pesquisa é, portanto, uma colaboração para o monitoramento da espécie em extinção Jacaratia spinosa (Aubl.) A.DC e dos fragmentos florestais em que estão localizadas no norte do estado do Paraná, para que sejam preservados devido à sua importância e diversidade dos seus recursos.

\section{REFERÊNCIAS}

ANJOS, L. dos; BOÇON, R. Bird communities in natural forest patches in southern Brazil. The Wilson Bul, n. 111, p. 397-414, 1999.

BAKER, H. G. "Mistake" pollination as a reproductive system with special reference to the Caricaceae, p. 161-169. In: BURLEY, J.; STYLES, B. T. (Ed.). Tropical trees: variation, breeding and conservation. London: Academic Press, 1976.

BATTILANI, J. L.; DIAS, E. S.; SOUZA, A. L. T. Fitossociologia de um trecho da mata ciliar do rio da Prata, Jardim, MS, Brasil. Acta Botânica Brasílica, v. 19, n. 3, p. 597-608, 2005.

CERVIGNE, N. S. Composição e estrutura do componente arbóreo dos remanescentes florestais da Fazenda Solana, Arapongas/PR. Monografia (Graduação em Ciências Biológicas). 31 p. 2007.

CORREIA, P. Dicionário das plantas úteis do Brasil. Rio de Janeiro: Imprensa Nacional, 1984. v. 3, p. 545.

DITT, E. H. Fragmentos florestais no Pontal do Paranapanema. São Paulo: Annablume, 2002. 140 p.

FELFILI, J. M.; REZENDE, R. P. Conceitos e métodos em fitossociologia. Brasília: Universidade de Brasília/Departamento de Engenharia Florestal, 2003. 34 p.

INSTITUTO AGRONÔMICO DO PARANÁ (IAPAR). Cartas climáticas do estado do Paraná. Londrina, p. 9-11, 1994. 45 p.

INSTITUTO PARANAENSE DE DESENVOLVIMENTO ECONÔMICO E SOCIAL (IPARDES). Cobertura florestal e consumo de madeira, lenha e carvão nas microrregiões de Londrina, Maringá e Paranavaí. Curitiba, 1993.
KALLIOLA, R. et al. The dynamics, distribution and classification of swamp vegetation in Peruvian Amazonia. Ann. Bot. Fennici, n. 28, p. 225-239, 1988.

LIMA, L. R.; PIRANI, J. R. Caricaceae. In: WANDERLEY, M. G. L.; SHEPHERD, G. J.; GIULIETTI, A. M. Flora fanerogâmica do estado de São Paulo. São Paulo: FAPESP/HUCITEC, 2002. v. 2 , cap. 2 , p. $79-82$.

LORENZI, H. Árvores brasileiras: manual de identificação e cultivo de plantas arbóreas nativas do Brasil. São Paulo: Nova Odessa/Plantarum, 2002.

MENEZES, P. R.; NETTO, J. S. M. Sensoriamento remoto dos alvos naturais. Brasília, DF: UnB; Planaltina: Embrapa Cerrados, 2001. 262 p.

METZGER, J. P. Estrutura da paisagem: o uso adequado de métricas. In: JUNIOR, L. C., PÁDUA, C. V.; RUDRAN, R. Métodos de estudos em biologia da conservação e manejo da vida silvestre. Curitiba: Ed. UFPR/Fundação O Boticário de Proteção à Natureza, 2003. 667 p.

MORAES, L. F. D. et al. Plantio de espécies arbóreas nativas para a restauração ecológica na reserva biológica de Poço das Antas. Rio de Janeiro, v. 57, n. 3, p. 477-489, 2006.

MOREIRA, M. A. Fundamentos do sensoriamento remoto e metodologia de aplicação. São Jose dos Campos, 2005.

MUNIZ, F. A. M. et al. Processo econômico de obtenção de celulose e papel a partir de Jaracatiá Spinosa. Revista da Propriedade Industrial, Brasília, n. 1817, p. 94, nov. 2004.

PARANÁ. Secretaria de Estado do Meio Ambiente. Lista vermelha de plantas ameaçadas de extinção no estado do Paraná. Curitiba: SEMA/GTZ, 1995. 139 p. il.

RODERJAN, C. V. et al. As unidades fitogeográficas do estado do Paraná. Ciência \& Ambiente, Santa Maria, v. 13, n. 1, p. 75-92, 2002.

SANTOS, G. A. S. D. Dieta e dispersão de sementes pelo "Bugio ruivo" Allouata clamitans Cabrera, 1940 (Primates, Atelidae) em um fragmento florestal no Norte do Paraná, Brasil. 85 p. Dissertação (Mestrado em Ciências Biológicas) - Universidade Estadual de Londrina, 2007.

SEMA. Secretaria do Meio Ambiente do Estado do Rio Grande do Sul. Lista das espécies ameaçadas de extinção no RS. 2002. Disponível em: <http://www.sema.rs.gov.br/sema/html/ espec.htm>. Acesso em: 8/5/2007.

SILVA, L. A.; SOARES, J. J. Levantamento fitossociológico em um fragmento de floresta estacional semidecídua, no município de São Carlos, SP. Acta Botânica Brasílica, v. 16, n. 2, p. 205-216, 2002. 
